\title{
Generalized magnification in visual optics. Part 2: Magnification as affine transformation
}

\section{WF Harris}

Department of Optometry, University of Johannesburg, PO Box 524, Auckland Park, 2006 South Africa

$<$ wharris@uj.ac.za>

Received 15 April 2010; revised version accepted 17 November 2010

\begin{abstract}
In astigmatic systems magnification may be different in different directions. It may also be accompanied by rotation or reflection. These changes from object to image are examples of generalized magnification. They are represented by $2 \times 2$ matrices. Because they are linear transformations they can be called linear magnifications. Linear magnifications account for a change in appearance without regard to position. Mathematical structure suggests a natural further generalization to a magnification that is complete in the sense that it accounts
\end{abstract}

for change in appearance and position. It is represented by a $3 \times 3$ matrix with a dummy third row. The transformation is called affine in linear algebra which suggests that these generalized magnifications be called affine magnifications. The purpose of the paper is to define affine magnification in the context of astigmatic optics. Several examples are presented and illustrated graphically. (S Afr Optom 2010 69(4) 166-172)

Key words: Linear magnification, affine magnification, transverse translation, astigmatism

\section{Introduction}

In conventional usage magnification means a proportional increase in size that is equal in all directions. It is represented by a scalar. Transverse magnification, for example, in Gaussian optics, can be written $m h=h^{\prime}$.

The magnification $m$ magnifies the height $h$ of an object to the height $h^{\prime}$ of its image. In the presence of astigmatism, however, the transformation is usually much more complicated and may involve changes not usually described as magnification. There may be conventional magnification but it can be accompanied by other changes in form and orientation. The natural generalization of the concept of magnification in linear optics is provided by the equation $\mathbf{X x}=\mathbf{y}$.
The $2 \times 2$ real matrix $\mathbf{X}$, with its four entries, operates on the $2 \times 1$ matrix $\mathbf{x}$ to form the $2 \times 1$ matrix $\mathbf{y} . \mathbf{x}$ and $\mathbf{y}$ may be vectors with horizontal and vertical components. $\mathbf{X}$ magnifies $\mathbf{x}$ to $\mathbf{y}$ in a generalized sense and $\mathbf{X}$ is the generalized magnification. Generalized magnification in this sense was the topic of Part 1 of this paper ${ }^{1}$.

In the sense used before ${ }^{1}$ generalized magnification is equivalent to what is called a linear transformation in linear algebra and, so, is appropriately termed linear magnification. In a sense, though, this generalized magnification is not quite complete: it accounts for change in size, shape and orientation but it takes no account of change in position. The purpose of this part is to show that we can take advantage of the mathematical structure to complete the concept of 
magnification in a natural way so that it now accounts for changes in size, shape and orientation and for change in position as well. Magnification becomes generalized still further. In a process essentially the same as the expansion of the $4 \times 4$ transference to a $5 \times 5$ transference by the addition of a fifth row and fifth column ${ }^{2,3}$ the matrix representing the magnification expands from $2 \times 2$ to $3 \times 3$ with two additional entries in the third column and a dummy third row. The operation of magnification is equivalent to what is known as an affine transformation in linear algebra; accordingly we can distinguish it as affine magnification. We may also distinguish linear and affine magnifications as $2 \times 2$ and $3 \times 3$ magnifications.

How far can this process go? Could we find yet further useful generalizations? Towards the end of this paper we shall argue that the answer could be Yes or No. One hyper-generalization we know already; if we are willing to call it a magnification, then we answer Yes. But for most applications of interest in optometry the generalized magnification of the type treated here appears to be complete.

\section{Affine magnification}

An affine transformation transforms a vector $\mathbf{x}$ to a second vector $\mathbf{y}$ according to ${ }^{4}$

$\mathbf{X x}+\mathbf{d}=\mathbf{y}$

where $\mathbf{d}$ is a $2 \times 1$ real matrix. Consider the following $3 \times 3$ matrix:

$$
\mathbf{Y}=\left(\begin{array}{cc}
\mathbf{X} & \mathbf{d} \\
\mathbf{o}^{\mathrm{T}} & 1
\end{array}\right)
$$

where $\mathbf{X}$ and $\mathbf{d}$ are as defined. $\mathbf{o}$ is the $2 \times 1$ null vector and $\mathbf{0}^{\mathrm{T}}$ its matrix transpose. We think of $\mathbf{Y}$ as $\mathbf{X}$ augmented by a third column (containing $\mathbf{d}$ ) and a dummy bottom row. We also define the $3 \times 1$ matrices

$$
\mathbf{u}=\left(\begin{array}{l}
\mathbf{x} \\
1
\end{array}\right)
$$

and

$$
\mathbf{v}=\left(\begin{array}{l}
\mathbf{y} \\
1
\end{array}\right)
$$

If we perform the block product Yu we obtain two equations: Equation 3 and the trivial equation $1=1$. In fact we have

$\mathbf{Y u}=\mathbf{v}$.

In effect we have transformed Equation 3 into an equation, Equation 7, which looks very much like Equation 2.

We can think of matrix $\mathbf{Y}$ as a kind of magnification that magnifies $\mathbf{u}$ to $\mathbf{v}$. $\mathbf{u}$ and $\mathbf{v}$ are really just the original $\mathbf{x}$ and $\mathbf{y}$ augmented by a dummy 1 in the third row that is there only so that matrix multiplication works. $\mathbf{Y}$ is the generalized magnification $\mathbf{X}$ of Part 1 generalized still further; $\mathbf{Y}$ represents affine magnification, $\mathbf{X}$ linear magnification. What was said before ${ }^{1}$ still holds; what is new in affine magnification is vector $\mathbf{d}$ in the third column.

Before we look at the physical meaning of affine magnification we take a quick look at successive affine magnification.

\section{Successive affine magnification}

Consider two affine magnifications,

$$
\mathbf{Y}_{1}=\left(\begin{array}{cc}
\mathbf{X}_{1} & \mathbf{d}_{1} \\
\mathbf{o}^{\mathrm{T}} & 1
\end{array}\right)
$$

and $\mathbf{Y}_{2}$ defined similarly. Suppose a vector $\mathbf{u}$ is first subjected to magnification $\mathbf{Y}_{1}$ and the result to magnification $\mathbf{Y}_{2}$. According to Equation 7 the result of the first magnification is

$\mathbf{Y}_{1} \mathbf{u}=\mathbf{v}$

and, again according to Equation 7, the result of the second magnification is

$\mathbf{Y}_{2} \mathbf{v}=\mathbf{w}$.

Substituting from Equation 9 into Equation 10 we obtain

$\mathbf{Y}_{2} \mathbf{Y}_{1} \mathbf{u}=\mathbf{w}$.

We write this as

$\mathbf{Y u}=\mathbf{w}$

where

$\mathbf{Y}_{2} \mathbf{Y}_{1}=\mathbf{Y}$.

Equation 12 is Equation 7 again but applied to magnification of $\mathbf{u}$ to $\mathbf{w}$. It follows that successive affine magnification by $\mathbf{Y}_{1}$ and then by $\mathbf{Y}_{2}$ is equivalent to 
the single magnification $\mathbf{Y}$ defined by Equation 13 . With more successive magnifications one finds that

$$
\cdots \mathbf{Y}_{3} \mathbf{Y}_{2} \mathbf{Y}_{1}=\mathbf{Y} \text {. }
$$

This pattern of successive affine magnifications being represented as matrix multiplication in reverse repeats that for successive linear magnifications and echoes what happens with transferences of optical systems in order ${ }^{2,3}$.

Again, because matrix multiplication does not commute, one sees from Equation 14 that the order of affine magnifications matters.

\section{Linear magnifications as affine magnifications}

\section{Suppose}

$$
\mathbf{Y}=\left(\begin{array}{cc}
\mathbf{X} & \mathbf{0} \\
\mathbf{0} & 1
\end{array}\right)
$$

This represents an affine magnification with $\mathbf{d}=\mathbf{0}$. In this case Equation 7, and equivalent Equation 3, reduce to Equation 2. In other words affine magnifications that happen to have $\mathbf{d}=\mathbf{0}$ are linear magnifications. Linear magnifications are affine. The converse, however, is not true. Linear magnifications are a subset of affine magnifications.

Linear magnifications are responsible for a variety of magnifications, distortions, rotations, et cetera, as described $^{1}$ in Part 1 . They can be decomposed into successive components as described before ${ }^{1}$.

\section{Transverse translations as affine magnifications}

Suppose now that

$$
\mathbf{Y}=\left(\begin{array}{cc}
\mathbf{I} & \mathbf{d} \\
\mathbf{o}^{\mathrm{T}} & 1
\end{array}\right)
$$

Then

$$
\mathbf{X}=\mathbf{I}
$$

where $\mathbf{I}$ is an identity matrix. Equations 7 and 3 become

$$
\mathbf{x}+\mathbf{d}=\mathbf{y} \text {. }
$$

Thus magnification of this type merely adds a fixed vector $\mathbf{d}$ to every vector $\mathbf{x}$ to get vector $\mathbf{y}$. In other words all points get shifted sideways by vector d. More formally we say that the magnification is a transverse translation by $\mathbf{d}$.

In conventional usage a sideways displacement would not usually be regarded as a magnification. But it does represent a change in appearance of the image relative to the object and becomes a magnification in this generalized affine sense.

\section{Affine magnification as combination of linear mag- nification and translation}

$\mathbf{Y}$ of Equation 15 is a linear magnification and $\mathbf{Y}$ of Equation 16 is a transverse translation. One can think of them as basic affine magnifications.

Suppose a linear magnification is followed by a transverse translation. Then, according to Equation 13

$\left(\begin{array}{cc}\mathbf{I} & \mathbf{d} \\ \mathbf{o}^{\mathrm{T}} & 1\end{array}\right)\left(\begin{array}{cc}\mathbf{X} & \mathbf{0} \\ \mathbf{o}^{\mathrm{T}} & 1\end{array}\right)=\left(\begin{array}{cc}\mathbf{X} & \mathbf{d} \\ \mathbf{o}^{\mathrm{T}} & 1\end{array}\right)$.

If one reverses the order then one finds that there is a change in the third column:

$\left(\begin{array}{cc}\mathbf{X} & \mathbf{o} \\ \mathbf{o}^{\mathrm{T}} & 1\end{array}\right)\left(\begin{array}{cc}\mathbf{I} & \mathbf{d} \\ \mathbf{o}^{\mathrm{T}} & 1\end{array}\right)=\left(\begin{array}{cc}\mathbf{X} & \mathbf{X d} \\ \mathbf{o}^{\mathrm{T}} & 1\end{array}\right)$

In the light of Equation 13 Equation 19 tells us that we can interpret any affine magnification $\left(\begin{array}{cc}\mathbf{X} & \mathbf{d} \\ \mathbf{o}^{\mathrm{T}} & 1\end{array}\right)$ as a linear magnification $\mathbf{X}$ followed by a transverse translation $\mathbf{d}$. Linear transformation, treated in detail in Part $1^{1}$, changes shape, size and orientation. By packaging transverse translation $\mathbf{d}$ into affine magnification $\mathbf{Y}$, together with linear magnification $\mathbf{X}$, we are now thinking of the transverse translation as part of the generalized magnification.

If we want to interpret $\left(\begin{array}{cc}\mathbf{X} & \mathbf{d} \\ \mathbf{o}^{\mathrm{T}} & 1\end{array}\right)$ as a transverse translation followed by a linear magnification then Equation 20 shows that the linear magnification would again be $\mathbf{X}$ but the transverse translation would have d replaced by

$\mathbf{d}^{\prime}=\mathbf{X}^{-1} \mathbf{d}$.

This interpretation is possible if the linear magnification $\mathbf{X}$ is nonsingular. If $\mathbf{X}$ is singular one has no option but to interpret transverse translation as occurring after linear magnification. 


\section{Some examples}

We consider here some specific examples of affine magnification. We begin with some special affine magnifications.

The magnification

$$
\mathbf{Y}_{1}=\left(\begin{array}{cc}
\mathbf{O} & \mathbf{0} \\
\mathbf{o}^{\mathrm{T}} & 1
\end{array}\right)
$$

operates on $\mathbf{u}=\left(\begin{array}{l}\mathbf{x} \\ 1\end{array}\right)$ to produce $\mathbf{v}=\left(\begin{array}{l}\mathbf{0} \\ 1\end{array}\right)$. There is linear magnification to nothing followed by no transverse translation. In other words every vector $\mathbf{x}$ is reduced to the null vector $(\mathbf{y}=\mathbf{0})$ as shown in Figure 1(a). The same holds for every point of an object.

Thus affine magnification by $\mathbf{Y}_{1}$ reduces all objects to an image consisting of a single point at the origin as in Figure 1(b).
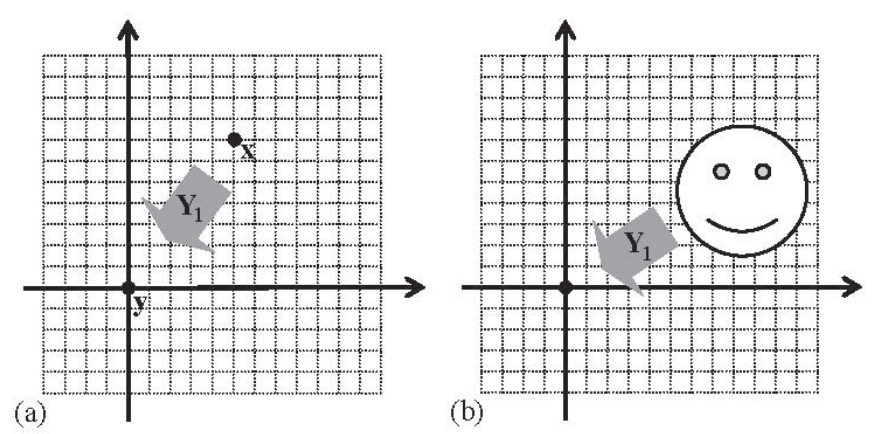

Figure 1 Affine magnification by $\mathbf{Y}_{1}$ defined by Equation 22 . (a) Vector $\mathbf{x}$ is reduced to a null vector. (b) The same happens to all points of the object resulting in the image being a point at the origin.

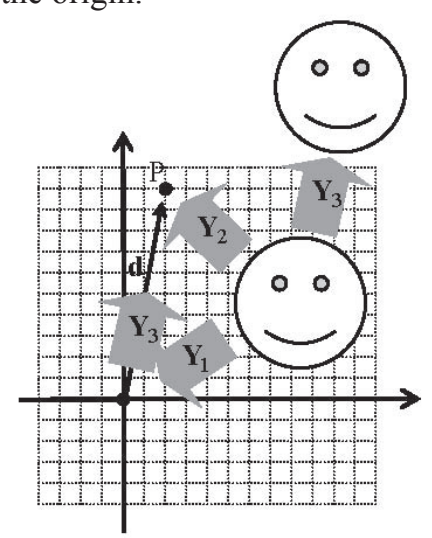

Figure 2 Affine magnification by $\mathbf{Y}_{2}$ defined by Equation 23. The image of the object is the point at $\mathrm{P} . \mathbf{Y}_{2}$ is equivalent to affine magnification by $\mathbf{Y}_{1}$ followed by affine magnification $\mathbf{Y}_{3}$ which is merely translation by $\mathbf{d}$. If $\mathbf{Y}_{3}$ acts first then the object is displace by $\mathbf{d}$ without change in shape, size or orientation; $\mathbf{Y}_{1}$ then shrinks the object to a point at the origin.
The affine magnification

$$
\mathbf{Y}_{2}=\left(\begin{array}{ccc}
0 & 0 & 2 \\
0 & 0 & 10 \\
0 & 0 & 1
\end{array}\right)
$$

differs from $\mathbf{Y}_{1}$ in that instead of being null $\mathbf{d}$ is now $\mathbf{d}=\left(\begin{array}{c}2 \\ 10\end{array}\right)$.

$\mathbf{d}$ is shown in Figure 2. Affine magnification by $\mathbf{Y}_{2}$ can be thought of as null linear magnification followed by translation d (Figure 2). In effect it reduces the object to a point at the origin and then displaces the point by $\mathbf{d}$. $\mathbf{Y}_{2}$, then, is $\mathbf{Y}_{1}$ followed by

$$
\mathbf{Y}_{3}=\left(\begin{array}{ccc}
1 & 0 & 2 \\
0 & 1 & 10 \\
0 & 0 & 1
\end{array}\right) \text {. }
$$

(The reader might wish to confirm that $\mathbf{Y}_{3} \mathbf{Y}_{1}=\mathbf{Y}_{2}$.)

Notice the difference if we reverse the order from $\mathbf{Y}_{3} \mathbf{Y}_{1}$ to $\mathbf{Y}_{1} \mathbf{Y}_{3}$. Affine magnification by $\mathbf{Y}_{3}$ is a transverse displacement of the object up and to the right by d as shown in Figure 2. Because the linear magnification involved in that step is $\mathbf{X}=\mathbf{I}$ (it is identity linear magnification), there is no change in shape, size or orientation. Then affine magnification by $\mathbf{Y}_{1}$ reduces everything to a point at the origin. Thus $\mathbf{Y}_{1} \mathbf{Y}_{3}=\mathbf{Y}_{1}$.

Consider the affine magnification

$\mathbf{Y}_{4}=\left(\begin{array}{ccc}1 & 3 & 1 \\ 1 & -1 & 3 \\ 0 & 0 & 1\end{array}\right)$

The top-left linear block

$\mathbf{X}_{4}=\left(\begin{array}{cc}1 & 3 \\ 1 & -1\end{array}\right)$

represents the same linear magnification illustrated in Figure 2 of Part 11. Figure 3 illustrates the effect of $\mathbf{Y}_{4}$ in converting object $\mathrm{O}$ to image $\mathrm{I}$. It can be interpreted as linear magnification by $\mathbf{X}_{4}$ followed by transverse translation

$\mathbf{d}_{4}=\left(\begin{array}{l}1 \\ 3\end{array}\right)$

or, equivalently, affine magnification by

$\mathbf{Y}_{5}=\left(\begin{array}{cc}\mathbf{X}_{4} & \mathbf{o} \\ \mathbf{o}^{\mathrm{T}} & 1\end{array}\right)$

followed by affine magnification 


$$
\mathbf{Y}_{6}=\left(\begin{array}{cc}
\mathbf{I} & \mathbf{d}_{4} \\
\mathbf{o}^{\mathrm{T}} & 1
\end{array}\right) \text {. }
$$

The steps are illustrated in Figure 3. $\mathbf{Y}_{5}$ magnifies object $\mathrm{O}$ to the intermediate image $\mathrm{I}_{1}$ represented by the dashed line and then $\mathbf{Y}_{6}$ displaces the result by $\mathbf{d}_{4}$.

Because $\mathbf{X}_{4}$ is nonsingular it is also possible to regard $\mathbf{Y}_{4}$ as a transverse translation followed by a linear magnification. According to Equation 21 the transverse translation is

$\mathbf{Y}_{7}=\left(\begin{array}{cc}\mathbf{I} & \mathbf{d}_{4}^{\prime} \\ \mathbf{o}^{\mathrm{T}} & 1\end{array}\right)$

where

$\mathbf{d}_{4}^{\prime}=\left(\begin{array}{c}2.5 \\ -0.5\end{array}\right)$.

The linear magnification is $\mathbf{Y}_{5}$. The first step results in intermediate image $\mathrm{I}_{2}$ (dotted in Figure 3); and the second step converts $\mathrm{I}_{2}$ to I. Figure 3 illustrates the fact that the translations involved are not generally the same if the order of the operations is reversed.

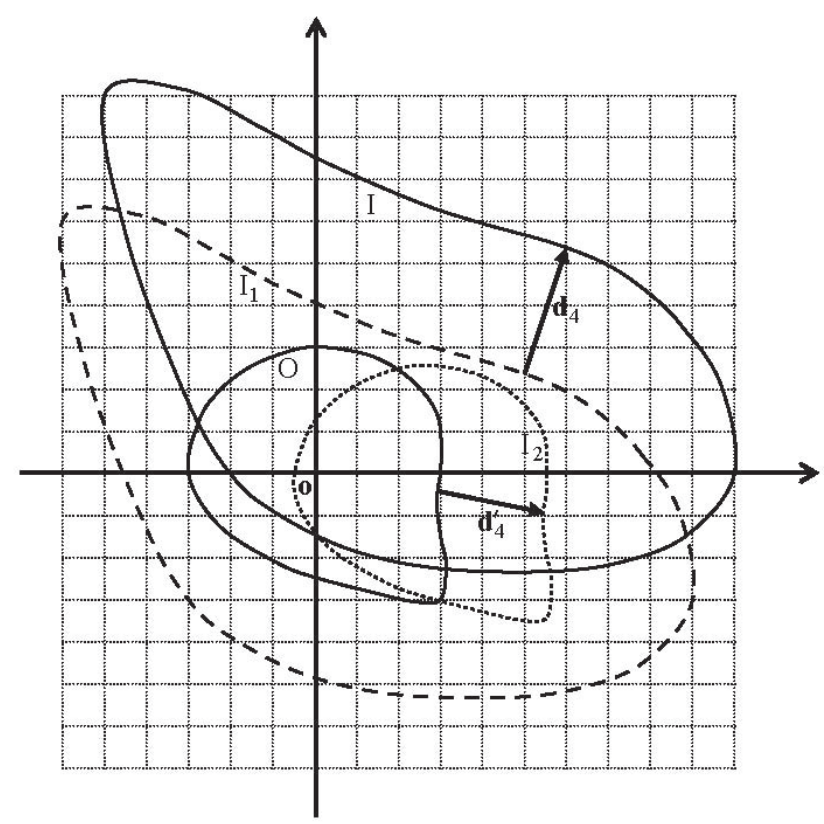

Figure 3 Affine magnification of object $\mathrm{O}$ by $\mathbf{Y}_{4}$ (Equation 26) to image I. It is equivalent to magnification by $\mathbf{Y}_{5}$ (Equation 29) to intermediate image $I_{1}$ followed by magnification $\mathbf{Y}_{6}$ (Equation 30) to I. It is also equivalent to magnification by $\mathbf{Y}_{7}$ (Equation 31 ) to $I_{2}$ followed by magnification by $\mathbf{Y}_{5}$ to $I$.

\section{Magnification and transference}

Before concluding we briefly comment on the relationship between generalized magnification and the ray transference.

The basic equation of linear optics is ${ }^{1-3}$

$\mathbf{T} \gamma_{0}=\gamma$

where $\mathbf{T}$ is the $5 \times 5$ transference of a system. $\gamma_{0}$ and $\gamma$ represent the state of a ray at incidence onto and emergence from the system respectively. The matrices have dummy bottom rows as shown explicitly when Equation 33 is written in the form

$\left(\begin{array}{cc}\mathbf{S} & \boldsymbol{\delta} \\ \mathbf{o}^{\mathrm{T}} & 1\end{array}\right)\left(\begin{array}{c}\boldsymbol{\rho}_{0} \\ 1\end{array}\right)=\left(\begin{array}{c}\boldsymbol{\rho} \\ 1\end{array}\right)$.

These two equations have the same forms as in Equations 7 and 4 respectively. So one can regard $\mathbf{T}$ as an affine magnification of the states of rays across the system and think of it as linear magnification $\mathbf{S}$ of the states of the rays followed by their transverse translation by $\delta$. The displacements are in a four-dimensional space, however, as opposed to the two-dimensional displacements we have been talking about above.

Equation 34 is often split into two equations

$\mathbf{A \mathbf { y } _ { 0 }}+\mathbf{B} \boldsymbol{\alpha}_{0}+\mathbf{e}=\mathbf{y}$

and

$\mathrm{Cy}_{0}+\mathbf{D} \boldsymbol{\alpha}_{0}+\pi=\alpha$.

Suppose we are dealing with a situation in which all rays entering the system are parallel. Then $\boldsymbol{\alpha}_{0}$ is constant. Further $\mathbf{B} \boldsymbol{\alpha}_{0}+\mathbf{e}$ is a constant $2 \times 1$ matrix. Equation 34 can, therefore, be written as

$\left(\begin{array}{cc}\mathbf{A} & \mathbf{B} \boldsymbol{\alpha}_{0}+\mathbf{e} \\ \mathbf{o}^{\mathrm{T}} & 1\end{array}\right)\left(\begin{array}{c}\mathbf{y}_{0} \\ 1\end{array}\right)=\left(\begin{array}{l}\mathbf{y} \\ 1\end{array}\right)$.

Thus

$\mathbf{Y}_{\mathbf{A}}=\left(\begin{array}{cc}\mathbf{A} & \mathbf{B} \boldsymbol{\alpha}_{0}+\mathbf{e} \\ \mathbf{o}^{\mathrm{T}} & 1\end{array}\right)$

represents an affine magnification of the transverse positions of parallel incident rays to their emergent positions. It would apply, for example, to an eye of ametropia $\mathbf{A}$ viewing a distant point object and relate positions on the cornea to the shape, size, orientation and location of the blur patch on the retina. Then $\mathbf{Y}_{\mathbf{A}}$ would be a kind of augmented ametropia $\mathbf{A}$ that characterizes image blur and location.

Similarly one can also write

$$
\left(\begin{array}{cc}
\mathbf{B} & \mathbf{A \mathbf { y } _ { 0 }}+\mathbf{e} \\
\mathbf{o}^{\mathrm{T}} & 1
\end{array}\right)\left(\begin{array}{c}
\boldsymbol{\alpha}_{0} \\
1
\end{array}\right)=\left(\begin{array}{l}
\mathbf{y} \\
1
\end{array}\right)
$$




$$
\left(\begin{array}{cc}
\mathbf{C} & \mathbf{D} \boldsymbol{\alpha}_{0}+\boldsymbol{\pi} \\
\mathbf{o}^{\mathrm{T}} & 1
\end{array}\right)\left(\begin{array}{c}
\mathbf{y}_{0} \\
1
\end{array}\right)=\left(\begin{array}{c}
\boldsymbol{\alpha} \\
1
\end{array}\right)
$$

and

$$
\left(\begin{array}{cc}
\mathbf{D} & \mathbf{C} \mathbf{y}_{0}+\pi \\
\mathbf{o}^{\mathrm{T}} & 1
\end{array}\right)\left(\begin{array}{c}
\boldsymbol{\alpha}_{0} \\
1
\end{array}\right)=\left(\begin{array}{c}
\boldsymbol{\alpha} \\
1
\end{array}\right) .
$$

Thus we have an affine magnification defined for each of the fundamental properties $\mathbf{A}, \mathbf{B}, \mathbf{C}$ and $\mathbf{D}$. They are augmented forms of the fundamental properties. Equation 41 represents affine magnification of reduced inclination of rays across the system. Equations 39 and 40 represent cross magnifications from reduced inclination to transverse position and transverse position to reduced inclination respectively. Equation 40 is, in fact, a further generalization of Imbert's equation ${ }^{5}$, the equation commonly called Prentice's equation ${ }^{5-7}$.

The four augmented fundamental properties $\mathbf{Y}_{\mathbf{A}}$, $\mathbf{Y}_{\mathbf{B}}, \mathbf{Y}_{\mathbf{C}}$ and $\mathbf{Y}_{\mathbf{D}}$ are affine magnifications of aspects of the state of a ray across a system. In a sense they are all wrapped up in the global affine magnification, the transference $\mathbf{T}$, which represents affine magnification of the state of the ray as a whole.

While it may be useful to think in terms of $5 \times 5$ affine magnifications like $\mathbf{T}$, and, perhaps, even those with more rows and columns, many optometric applications concern mappings between surfaces: object plane, corneal plane, iridial plane and retinal plane. In such cases $3 \times 3$ affine magnifications are as far as one need to go. And they are complete because they account for change in form, size, orientation and location of image in two dimensions.

\section{Concluding remarks}

Magnification, as generalized in Part $1^{1}$, is simply a linear transformation. It is represented by a $2 \times 2$ matrix $\mathbf{X}$. Its effect is to change the appearance of the object without regard to its position. The resulting image may be magnified, perhaps differently in different meridians, and rotated or reflected. Here we generalize further to a magnification represented by a $3 \times 3$ matrix $\mathbf{Y}$ of which $\mathbf{X}$ is a submatrix according to Equation 4. There is also a second submatrix $\mathbf{d}$. In generalizing from linear magnification to affine magnification we are incorporating position of the image into our concept of magnification as well. Thus affine magnification implies a change in appearance including position.

Whether one uses linear or affine magnification depends on the application. One may be interested only in the form of the image and not its location. In that case linear magnification is appropriate and it is unnecessary to use affine magnification.

Affine magnification can be decomposed into linear magnification $\mathbf{X}$ followed by transverse translation by vector $\mathbf{d}$. If $\mathbf{X}$ is not singular one can reverse the order of the component operations but then translation is different; it is $\mathbf{d}^{\prime}$ given by Equation 21.

There is nothing new here in the mathematics, and there is nothing here that cannot be done without the concept of affine magnification. What the concept does, however, is that it takes advantage of the extraordinarily compact nature of linear algebra by packing positional changes, as it were, into the same parcel as shape, size and orientation. In doing so it streamlines Equation 3 into the elegantly simple Equation 7 which looks the same as so many other equations. Instead of two parcels, $\mathbf{X}$ and $\mathbf{d}$, we have to keep track of only one, Y. At first sight this might not seem much of an advantage; but if one sets out, acquiring double the number of parcels necessary at each stop, one quickly gets the point. And because the many different packages all assume the same standard mathematical form we have the possibility of applying in new areas mathematical and statistical machinery developed for other applications. One must stress, however, that it is possibility not certainty; for one needs to remain cognisant of any special characteristics of particular magnifications such as the symplecticity ${ }^{8}$ of submatrix $\mathbf{S}$ within transference $\mathbf{T}$.

\section{Acknowledgements}

I gratefully acknowledge support from the National Research Foundation. I thank J R Cardoso and A Rubin for continuing discussions.

\section{References}

1. Harris WF. Generalized magnification in visual optics. Part 1: Magnification as linear transformation. S Afr Optom 2010 69 109-122. 
2. Harris WF. Paraxial ray tracing through noncoaxial astigmatic optical systems, and a $5 \times 5$ augmented system matrix. Optom Vis Sci 199471 282-285.

3. Harris WF. Flipped optical systems. Ophthal Physiol Opt 200828 595-602.

4. Bernstein DS. Matrix Mathematics: Theory, Facts, and Formulas. 2nd ed. Princeton University Press, Princeton 2009 88.

5. Imbert A. Calcul de l'effet prismatique des verres décentrés. Annal Oculist 188695 146-159.

6. Bennett AG. Prismatic effects of spherical and astigmatic lenses: Imbert's pioneer analysis of 1886. Ophthal Physiol Opt $199010397-398$.

7. Harris WF. Effect of spectacle and contact lenses on the effective corneal refractive zone. Clin Exper Optom 200992 99-103.

8. Harris WF. Symplecticity and relationships among the fundamental properties in linear optics. S Afr Optom 201069 $3-13$. 